\title{
Erratum: Competition between Covalent Bonding and Charge Transfer at Complex-Oxide Interfaces [Phys. Rev. Lett. 112, 196802 (2014)]
}

Juan Salafranca, Julián Rincón, Javier Tornos, Carlos León, Jacobo Santamaria, Elbio Dagotto,

Stephen J. Pennycook, and Maria Varela

(Received 30 September 2014; published 31 October 2014)

DOI: 10.1103/PhysRevLett.113.189902

PACS numbers: 73.20.-r, 74.20.-z, 74.78.Fk, 99.10.Cd

We have noticed an error in the inset of Fig. 2(a) of our Letter. While editing and preparing the figure for submission, the inset was corrupted and a random coloring was generated, which does not correspond to any actual EELS data set (as described in the figure caption). The correct inset is displayed in the figure shown here. This unfortunate error does not affect the text, caption, findings or conclusions of the manuscript in any way.
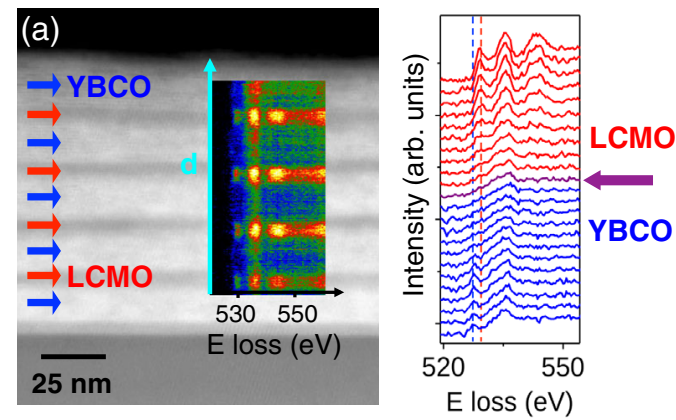

(b)

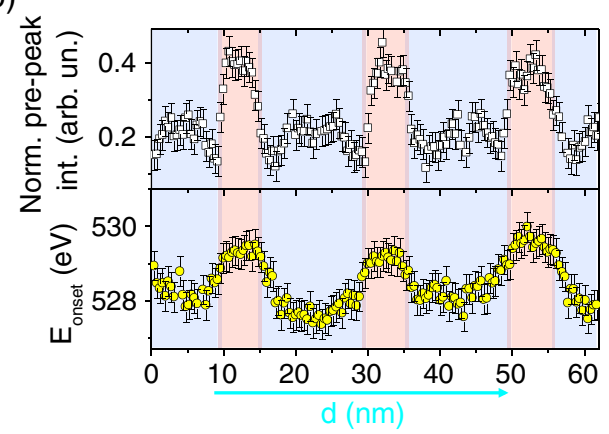

FIG. 2 (color online). (a) Z-contrast image of a LCMO/YBCO multilayer on a (100) $\mathrm{SrTiO}_{3}$ substrate. Arrows mark LCMO (red) and YBCO layers (blue). The inset shows an EELS linescan acquired along the growth direction. The right panel shows the energy range corresponding to the $\mathrm{O} K$ edge across one of the YBCO-LCMO interfaces in the line scan. Dashed lines marked the position of the $\mathrm{O}$ prepeak for LCMO and YBCO away from the interface. (b) Prepeak intensity (top), and the edge onset position (bottom) along the growth direction, marked with a light blue arrow. Some data adapted from previous work (see Supplemental Material [33] for details) 